Pacific Journal of Mathematics

ANALYTIC STRUCTURE IN THE SPECTRUM OF A NATURAL 


\title{
ANALYTIC STRUCTURE IN THE SPECTRUM OF A NATURAL SYSTEM
}

\author{
R. M. Brooks
}

\begin{abstract}
Let $[X, A]$ be a natural system such that $X$ is locally compact and every open subset of $X$ is $\sigma$-compact. Let $\mathscr{A}$ be the sheaf on $\mathbf{X}$ generated by the presheaf $\left\{U \rightarrow A_{U} \equiv \overline{A \mid U}\right\}$. If $p \in X, V$ is a subvariety of an open set in $C^{n}$ which contains 0 and if there exists an algebra homomorphism $\varphi: \mathscr{A}_{p} \rightarrow{ }_{v} \mathcal{O}_{0}$ having rank greater than one, then there exists a neighborhood $U$ of $p$ in $X$, a neighborhood $\omega$ of 0 in $C^{n}$ and a continuous map $\tau: V \cap \omega \rightarrow U$ such that $(1) \tau(0)=p$, (2) if $f \in A_{U}$, then $f \circ \tau$ is holomorphic on $V \cap \omega$, and $(3)$ if $f \in A_{U}$, then $(f \circ \tau)_{0}=\varphi\left((f)_{p}\right)$, where $(f \circ \tau)_{0}$ is the germ at 0 of $f \circ \tau$ and $(f)_{p}$ is the element of $\mathscr{A}_{p}$ (the stalk of $\mathscr{A}$ above $p$ ) determined by $f$.
\end{abstract}

The theorem quoted above is the main result of the paper. The crucial steps leading to it are Theorems 1.6,1.7, and 1.8, successive generalizations of a theorem of B. E. Johnson [9, Theorem 9.3], who considered the $n=1$ case of Theorem 1.6, and Theorem 2.12. Previous work closely related to the results of this paper has been done by Carpenter [2] ( $F$-algebras and $n=1)$, Clayton [3, 4] ( $B$-algebras and maps into ${ }_{V} O_{0}$ ), and Loy [10] ( $F$-algebras and special maps into $\left.{ }_{n} \mathcal{O}_{0}(n \geqq 1)\right)$. Each of the three used a sheaf somewhat different from the one used in this paper. We shall discuss the question of "which sheaf to use" at the end of $\S 3$.

1. On the continuity of certain linear maps. In this section we obtain the crucial results of the paper, to be applied in the next section to the problem under study. We begin with some notation and basic results on spaces of formal power series.

We denote by ${ }_{n} F$ the algebra of formal power series (over $C$ ) in $n$ variables $X_{1}, \cdots, X_{n}$. A typical element of ${ }_{n} F$ is a formal series $\sum_{J} a_{J} X^{J}$, where $J$ runs through $N^{n}, J=\left(j_{1}, \cdots, j_{n}\right)$ with each $j_{i} \in N$, and $X^{J}=X_{1}^{j_{i}}, \cdots, X_{n}^{j_{n}}$. For each $J \in \mathbb{N}^{n}$ we define a linear functional $p_{J}:{ }_{n} F \rightarrow C$ by $p_{J}\left(\sum_{I} a_{I} X^{I}\right) \equiv \alpha_{J}$. We endow ${ }_{n} F$ with the coarsest locally convex topology with respect to which each $p_{J}$ is continuous; i.e., the one determined by the family $\left\{\left|p_{J}\right|: J \in N^{n}\right\}$ of seminorms. This topology will be referred to below as the ${ }_{n} F$-topology.

Let $F$ be a subspace of ${ }_{n} F$ equipped with the ${ }_{n} F$-topology and let $M$ be a (relatively) closed subspace of $F$. Then $\left(F / M\right.$, quot $\left.\left({ }_{n} F\right)\right)$ is a separated locally convex LTS (see [8, p. 105]). The quotient topology on $F / M$ is determined by the family $\left\{p_{J}: J \in N^{n}\right\}$ as follows. For each $J \in \mathbb{N}^{n}$ we define $\left|p_{J}\right|^{\wedge}$ on $F / M$ by $\left|p_{J}\right|^{\wedge}([y])=\inf \left\{\left|p_{J}\right|(y+\right.$ 
$m): m \in \boldsymbol{M}\}=\inf \left\{\left|p_{J}(y+m)\right|: m \in M\right\}=\inf \left\{\left|p_{J}(y)+p_{J}(m)\right|: m \in M\right\}$. Thus, unless $p_{J} \equiv 0$ on $M$ the seminorm $\left|p_{J}\right|^{\wedge}$ is identically zero on $F / M$. Hence, the topology quot $\left({ }_{n} F\right)$ is determined by the subfamily $\left\{\hat{p}_{J}: J \in\left(N^{n}\right)_{M}\right\}$ consisting of those linear functionals which can be defined on $F / M,\left(\boldsymbol{N}^{n}\right)_{M}$ being the set of those $J \in \boldsymbol{N}^{n}$ such that $p_{J} \equiv 0$ on $M$ and $\hat{p}_{J}$ being the induced map on $F / M$ to $C$.

Definition. A locally convex $T V S(F, \mathscr{T})$ is called an $L C-K^{n}$ space provided that $F \subseteq{ }_{n} F$ and each of the maps $p_{J} \mid F$ is $\mathscr{T}$-continuous. An $L C-K^{n}$ space $F$ will be called an $L C(E q)-K^{n}$ space if there exists a family $\left\{C_{J}: J \in \boldsymbol{N}^{n}\right\} \subseteq \boldsymbol{R}^{+}$such that the family $\left\{C_{J}^{-1} p_{J}: J \in \boldsymbol{N}^{n}\right\}$ is equicontinuous (relative to the topology $\mathscr{T}$ on $F$ ). If $F$ is an $L C-K^{n}$ space we shall refer to the two topologies on $F$ as the " $F$ topology" and the " ${ }_{n} F$-topology".

Definition. Let $E$ and $F$ be $L C T V S$ 's. We shall say that “ $[E, F]$ is a $C G$-pair" provided that every linear map from $E$ to $F$ which has a closed graph is continuous.

REMARK. If $E$ is an inductive limit of $F$-spaces and $F$ is a countable inductive limit of $F$-spaces, then $[E, F]$ is a $C G$-pair (see [5, Theorem 6.7.1]).

Definition. Let $F$ be an $L C-K^{n}$ space. If $T: F \rightarrow F$ is a linear map, then for each $y \in F$ we have $T y \in F \subseteq{ }_{n} F$; hence, $p_{K}(T y)=$ $\sum_{J} a_{K J} p_{J}(y)$ for some family $\left(a_{K J}\right)$ of complex numbers. If $a_{K J} \neq 0$ only when $J \leqq K\left(j_{1} \leqq k_{1}, \cdots, j_{n} \leqq k_{n}\right)$, we shall call $T$ a lower triangular operator on $F$.

LEMMA 1.1. Let $E$ be an LCTVS and $F$ an $L C-K^{n}$ space such that $[E, F]$ is a CG-pair. If $u: E \rightarrow\left(F,{ }_{n} F\right.$-topology $)$ is continuous, then $u: E \rightarrow(F, F$-topology $)$ is continuous.

Proof. Since the ${ }_{n} F$-topology is separated it is easily proved that the graph of $u$ is closed.

Lemma 1.2. If $F$ is an $L C-K^{n}$ space such that $[F, F]$ is a $C G$ pair, then every lower triangular operator on $F$ is continuous.

Proof. It suffices to show that if $T$ is a lower triangular operator on $F$, then $p_{K} \circ T$ is continuous for each $K \in N^{n}$ (Lemma 1.1). Now $p_{k} \circ T(y)=\sum_{J \leqq K} a_{K J} p_{J}(y)$. This is clearly continuous, since each $p_{J}$ is continuous and the sum is finite.

Lemma 1.3. (Johnson [9, Theorem 7.3].) Suppose $[E, F]$ is a 
CG-pair of LCTVS's $\left\{G_{i}\right\}_{i \in I}$ is a family of LCTVS's, $u: E \rightarrow F$ is a linear map and for each $i w_{i}: F \rightarrow G_{i}$ is a continuous linear map. If for each $i \in I$ the map $w_{i} \circ u$ is continuous, then $\pi \circ u: E \rightarrow F / N$ is continuous, where $N=\cap\left\{\operatorname{Ker} w_{i}: i \in I\right\}$ and $\pi: F \rightarrow F / N$ is the natural projection.

\section{Proof. See [9].}

A useful special case of this lemma is

Corollary 1.4. Suppose $[E, F]$ is a CG-pair of LCTVS's. If $u: E \rightarrow F$ is a linear map, $w: F \rightarrow F$ is a continuous, linear injection, and if $w \circ u$ is continuous, then $u$ is continuous.

For easy reference we state Theorem 6.5.1 of [5]:

LEMmA 1.5. Let $E,\left\{F_{n}\right\}_{n=1}^{\infty}$ be $F$-spaces and let $F$ be a separated LCTVS. Suppose u: $E \rightarrow F, v_{n}: F_{n} \rightarrow F(n \in N)$ are continuous linear maps. If $u(E) \subseteq \bigcup_{n=1}^{\infty} v_{n}\left(F_{n}\right)$, then there exists $m \in N$ such that $u(E) \leqq v_{m}\left(F_{m}\right)$ and if $W_{m}$ is a neighborhood of 0 in $F_{m}$, then $u^{-1}\left(v_{m}\left(W_{n}\right)\right)$ is a neighborhood of 0 in $E$. In particular, if each $v_{n}$ is injective, then $v_{m}^{-1} \circ u: E \rightarrow F_{m}$ is continuous.

Our first main result is an extension of Johnson's Theorem 9.1 (see [9]) from the $n=1$ case to arbitrary finite $n$.

Theorem 1.6. Let $E$ be an $F$-space and $F$ an $L C(E q)-K^{n}$ space such that $[E, F]$ and $[F, F]$ are $C G$-pairs. Suppose (1) $S: E \rightarrow E$ is a continuous linear map and (2) $T: F \rightarrow F$ is a lower triangular linear map with empty point spectrum. If $u: E \rightarrow F$ is a linear map such that $u S=T u$, then $u$ is continuous.

Proof. We begin by observing that if $P$ is any monic polynomial with complex coefficients, then $P(T)$ is injective. We can write $P(T)=\prod_{j=1}^{m}\left(T-\alpha_{j} I\right)$. If $P(T) y=0$, then $\left(T-\alpha_{m} I\right)\left(\prod_{j=1}^{m-1}\left(T-\alpha_{j} I\right) y\right)=0$. Since the point spectrum of $T$ is empty we must have $\Pi_{j=1}^{m-1}\left(T-a_{j} I\right) y=0$. The argument can be completed by induction on the degree of $P$.

We let $\left(a_{K J}\right)$ denote the "lower triangular" matrix corresponding to $T$ and we define $Q_{K}: E \rightarrow E$ and $R_{K}: F \rightarrow F\left(K \in N^{n}\right)$

$$
\begin{aligned}
& Q_{K}=\prod_{J \leqq K}\left(S-a_{J J} I\right) \\
& R_{K}=\prod_{J \leqq K}\left(T-a_{J J} I\right) .
\end{aligned}
$$


If $J \leqq K$ we set $Q_{K \backslash J}=\Pi_{I \leqq K, I \nsubseteq J}\left(S-a_{I I} I\right)$ so that we have $Q_{K}=Q_{J} \circ Q_{K \backslash J}$. One defines $R_{K \backslash J}$ similarly. We note that (1) $u Q_{K}=R_{K} u$ for each $K \in N^{n}$ and (2) each $R_{K}$ is injective (since it is a monic polynomial in $T)$. Fix $K \in N^{n}, y \in F$. Then $p_{K}(T y)=\sum_{J \leqq K} a_{K J} p_{J}(y)$. Hence, $p_{K}\left(\left(T-a_{K K} I\right) y\right)=\sum_{J<K} a_{K J} p_{J}(y)$.

We next show that if $J \leqq K$, then $p_{J} \circ R_{K} \equiv 0$. We shall induct on $|K|\left(=k_{1}+\cdots+k_{n}\right)$. If $|K|=0$ and $J \leqq K$, then $J=K=(0$, $\cdots, 0)=\underline{0}$, and $p_{0}\left(R_{0} y\right)=p_{0}\left(\left(T-a_{00} I\right) y\right)=0$ since $T$ is lower triangular. Suppose we have established the claim whenever $|K|<q$. Fix $K \in N^{n}$ such that $|K|=q$ and $y \in F$. If $J<K$, then $p_{J}\left(R_{K} y\right)=$ $p_{J}\left(R_{J} R_{K \backslash J} y\right)=0$ since $|J|<q$. If $J=K$, then

$$
p_{K}\left(R_{K} y\right)=p_{K}\left(\left(T-a_{K K} I\right) R_{K}^{\prime} y\right),
$$

where $R_{K}^{\prime}=\Pi_{J<K}\left(T-a_{J J} I\right)$. Thus, $p_{K}\left(R_{K} y\right)=\sum_{J<K} a_{K J} p_{J}\left(R_{K}^{\prime} y\right)$. Now for each $J<K$ we have $p_{J}\left(R_{K}^{\prime} y\right)=p_{J}\left(R_{J} \circ R_{K \backslash J}^{\prime} y\right)$ where $R_{K \backslash J}^{\prime}=$ $\Pi_{I<K, I \$ J}\left(T-a_{I I} I\right)$. Hence, $p_{J}\left(R_{K}^{\prime} y\right)=0$ (again $J<K$ so $|J|<q$ ).

Since each $R_{K}$ is a continuous linear injection it is sufficient to find one $R_{K}$ such that $R_{K} u$ (equivalently, $u Q_{K}$ ) is continuous, (Corollary 1.4 above). In fact, either all the operators $R_{K} u$ are continuous or none are. Suppose $R_{K} u$ is continuous. If $J \leqq K$, then $R_{K}=R_{J} \circ R_{K \backslash J}$ and $R_{K} u=R_{K \backslash J} \circ R_{J} u$. Since $R_{K \backslash J}$ is injective we conclude that $R_{J} u$ is continuous. If $J \geqq K$, then $R_{J} u=R_{J \backslash K} \circ R_{K} u$, a composition of two continuous maps; hence $R_{J} u$ is continuous. Let $J \in N^{n}$ and let $L=$ $J+K$. Then $K \leqq L$ so $R_{L} u$ is continuous. But $J \leqq L$, so $R_{J} u$ is continuous.

We assume that no operator $R_{J} u$ is continuous; i.e., no operator $u Q_{J}$ is continuous. Hence, for each $J \in N^{n}$ there exists $K \in N^{n}$ such that $p_{K^{\circ}} \circ u Q_{J}$ is not continuous (Lemma 1.1 above). Set $J_{0}=(0, \cdots, 0)$, and fix $J_{1} \geqq J_{0}$. Choose $K_{1}$ such that $p_{K_{1}} \circ u Q_{J_{1}}$ is not continuous. Fix $J_{2} \geqq J_{1}, K_{1}$ and choose $K_{2}$ such that $p_{K_{2}} \circ u Q_{J_{2}}$ is not continuous. Continue the process inductively. Fix an increasing sequence $\left\{\|\cdot\|_{i}\right\}_{i=1}^{\infty}$ of seminorms for $E:\|x\|_{i} \leqq\|x\|_{i+1}(x \in E, i \in N)$. We choose inductively a sequence $\left\{x_{l}\right\}_{l=1}^{\infty}$ in $E$ such that for each $l \in N$

$$
\begin{gathered}
\left\|Q_{J_{l} \backslash J_{i}} x_{l}\right\|_{l}<2^{-l}(i=0,1, \cdots, l-1) . \\
\left|p_{K_{l}}\left(u Q_{J_{l}} x_{l}\right)\right|>l \cdot C_{K_{l}}+\sum_{i=1}^{l-1}\left|p_{K_{l}}\left(u Q_{J_{i}} x_{i}\right)\right|,
\end{gathered}
$$

where $\left\{C_{J}\right\}$ is the family of positive real numbers chosen so that $\left\{C_{J}^{-1} p_{J}\right\}$ is equicontinuous on $F$. We can make such a choice since each $Q_{J_{l} \backslash J_{i}}$ is continuous $(i=0,1, \cdots, l-1)$ and $p_{K_{l}} \circ u Q_{J_{l}}$ is not (hence, takes on arbitrary large absolute values on elements arbitrarily close to 0 in $E$ ). Let $x=\sum_{l=1}^{\infty} Q_{J_{l}} x_{l}$. The series converges absolutely in $E$ (condition (1) guarantees this). Fix $l \in N$. Then 


$$
\begin{aligned}
P_{K_{l}}(u x) & =p_{K_{l}}\left[\sum_{i \leqq l} u Q_{J_{i}} x_{i}+u\left(\sum_{i>l} Q_{J_{i}} x_{i}\right)\right] \\
& =p_{K_{l}}\left(\sum_{i \leqq l} u Q_{J_{i}} x_{i}\right)+p_{K_{l}}\left[u Q_{J_{l+1}}\left(\sum_{i>l} Q_{J_{i} \backslash J_{l+1}} x_{i}\right)\right] .
\end{aligned}
$$

This last step is possible because $Q_{J_{l+1}}$ is continuous and the series $\sum_{i>l} Q_{J_{i} \backslash J_{l+1}} x_{i}$ converges in $E:\left\|Q_{J_{i} \backslash J_{l+1}} x_{i}\right\|_{i}<2^{-i}(i>l)$. Now

$$
p_{K_{l}}\left(u Q_{J_{l+1}} x^{\prime}\right)=p_{K_{l}}\left(R_{J_{l+1}} u x^{\prime}\right)
$$

for $x^{\prime} \in E$. Since $K_{l} \leqq J_{l+1}$ this number is zero. Thus,

$$
\begin{aligned}
& p_{K_{l}}(u x)=\sum_{i=1}^{l} p_{K_{l}}\left(u Q_{J_{i}} x_{i}\right), \quad \text { so } \\
& \quad\left|p_{K_{l}}(u x)\right| \geqq\left|p_{K_{l}}\left(u Q_{J_{l}} x_{l}\right)\right|-\sum_{i=1}^{l-1}\left|p_{K_{l}}\left(u Q_{J_{i}} x_{i}\right)\right|
\end{aligned}
$$

and we have $\left|p_{K_{l}}(u x)\right|>l \cdot C_{K_{l}}$.

There exists a neighborhood $V$ of 0 in $F$ such that if $y \in V$ and $J \in N^{n}$, then $\left|p_{J}(y)\right|<C_{J}$. Since $u(x) \in F$ there exists $\lambda>0$ such that $\lambda u(x) \in V$. Hence, for each $l \in N$ we must have $\left|p_{K_{l}}(\lambda u(x))\right|<C_{K_{l}}$. Combining this with our previous inequality we obtain $l \lambda<1$ for each $l \in N$, a contradiction. Thus, $u Q_{J}$ is continuous for some $J \in N^{n}$ and $u$ is continuous.

Theorem 1.7. Let $E$ be an $F$-space and $F$ an $L C\left(E_{q}\right)-K^{n}$ space such that $[F, F]$ is a CG-pair. Let $M$ be a subspace of $F$ such that (1) $M$ is both $F$-and ${ }_{n} F$-closed and (2) $[E, F / M]$ is a CG-pair. Suppose (3) $S: E \rightarrow E$ is a continuous linear map and (4) $T: F \rightarrow F$ is a lower triangular operator on $F$ such that $T(M) \cong M$ and such that the induced map $\hat{T}: F / M \rightarrow F / M$ has empty point spectrum. If $u: E \rightarrow F / M$ is a linear map such that $u S=\hat{T} u$, then $u$ is continuous.

Proof. Let $\left(a_{K J}\right)$ be the lower triangular matrix corresponding to $T$. We define $Q_{K}, R_{K}$ as in the proof of Theorem 1.6 and define $\hat{R}_{K}$ on $F / M$ to be the map naturally induced by $R_{K}$, since $R_{K}(M) \subseteq M$. It is clear that $u Q_{K}=\hat{R}_{K} u$ for $K \in N^{n}$ and that each $\hat{R}_{K}$ is a monic polynomial in $\hat{T}$; hence, each $\hat{R}_{K}$ is injective. It is easily verified that $\hat{T}$ and the operators $\hat{R}_{K}$ are continuous on $F / M$, equipped with the quot $(F)$-topology. Recall that the quot $\left({ }_{n} F\right)$-topology on $F / M$ is determined by the linear maps $\left\{\hat{p}_{J}: J \in\left(N^{n}\right)_{M}\right\}$, where $\left(N^{n}\right)_{M}=\left\{J: p_{J} \equiv 0\right.$ on $M$. Since $[(E, F / M)]$ is a $C G$-pair we are in the same situation as in Theorem 1.6: we need only show that for one $J \in N^{n}$ the operator $\hat{R}_{J} u$ (equivalently, $u Q_{J}$ ) is continuous. Assume that none is. Let $J_{0}=(0, \cdots, 0)$. Fix $J_{1} \in N^{n}$. Choose $K_{1} \in\left(N^{n}\right)_{M}$ so that $\hat{p}_{K_{1}} \circ u Q_{J_{1}}$ is not continuous. Continue as in Theorem 1.6. The sequence $\left\{x_{l}\right\}_{l=1}^{\infty}$ is 
chosen as in that theorem (with $\hat{p}_{K_{l}}$ in place of $p_{K_{l}}$ ). The proof is completed as before, with two small problems to be resolved. We must show that (a) $\hat{p}_{K_{l}}\left[u Q_{J_{l+1}}\left(\sum_{i>l} Q_{J_{i} \backslash J l+1} x_{i}\right)\right]=0$ and (b) the family $\left\{C_{J}^{-1} \hat{p}_{J}: J \in\left(N^{n}\right)_{M}\right\}$ is equicontinuous on $F / M$.

Re (a). Let $x^{\prime}$ denote the sum in parentheses. We have $\hat{p}_{K_{l}}\left(u Q_{J_{l+1}} x^{\prime}\right)=\hat{p}_{K_{l}}\left(\left[\hat{R}_{J_{l+1}}\left(u x^{\prime}\right)\right]\right)$. Now $u\left(x^{\prime}\right)=\left[y^{\prime}\right]$ for some $y^{\prime} \in F$, and $\hat{p}_{K_{l}}\left(\hat{R}_{J_{l+1}}\left[y^{\prime}\right]\right)=\hat{p}_{K_{l}}\left(\left[R_{J_{l+1}} y^{\prime}\right]\right)=p_{K_{l}}\left(R_{J_{l+1}} y^{\prime}\right)=0$ (proved in Theorem 1.6), since $K_{l} \leqq J_{l+1}$.

Re (b). There exists a continuous seminorm $q$ on $F$ such that if $q(y)<1$ and $J \in N^{n}$, then $\left|p_{J}(y)\right|<C_{J}$. Let $\hat{q}$ be the corresponding seminorm on $F / M$. Fix $[y] \in F / M$ such that $\hat{q}([y])<1$ and $J \in\left(N^{n}\right)_{M}$. Then there exists $m \in M$ such that $q(y+m)<1$. Hence, $\left|p_{J}(y+m)\right|<C_{J}$, or $\left|p_{J}(y)+p_{J}(m)\right|<C_{J}$. However, $m \in M$ and $J \in\left(N^{n}\right)_{M}$. Thus $p_{J}(y)<C_{J}$ and $\left|\hat{p}_{J}([y])\right|<C_{J}$.

We now show that there is a large class of spaces $F$ for which our hypotheses regarding $C G$-pairs are satisfied.

Lemma 1.8. Let $E$ be an $F$-space and let $G$ be a vector space with two locally convex topologies $\mathscr{T}_{1}$ and $\mathscr{T}_{2}$, where $\mathscr{T}_{1} \leqq \mathscr{T}_{2}$. If $\left[E,\left(G, \mathscr{T}_{2}\right)\right]$ is a CG-pair, then so is $\left[E,\left(G, \mathscr{T}_{1}\right)\right]$.

Proof. Let $u: E \rightarrow G$ be a linear map such that $G r(u)$ is closed in $E \times\left(G, \mathscr{T}_{1}\right)$. If $\left\{\left(x_{n}, u\left(x_{n}\right)\right)\right\}$ is a sequence in $\operatorname{Gr}(u)$ which converges in $E \times\left(G, \mathscr{T}_{2}\right)$ to $(x, z)$, then $x_{n} \rightarrow x$ in $E$ and $u\left(x_{n}\right) \rightarrow z\left(\mathscr{T}_{2}\right)$. But $\mathscr{T}_{1} \leqq \mathscr{T}_{2}$, so $u\left(x_{n}\right) \rightarrow z\left(\mathscr{T}_{1}\right)$ and our hypotheses imply that $z=u(x)$. Hence, $G r(u)$ is closed in $E \times\left(G, \mathscr{T}_{2}\right)$ and $u$ is $\mathscr{T}_{2}$-continuous. Since $\mathscr{T}_{1} \leqq \mathscr{T}_{2}$ we have also that $u$ is $\mathscr{T}_{1}$-continuous.

Definition. An $L C$ space $(F, \mathscr{T})$ is an injective inductive limit of $F$-spaces provided there exists an increasing sequence $\left\{F_{n}\right\}_{n=1}^{\infty}$ of subspaces of $F$ such that (1) $F=\bigcup_{n=1}^{\infty} F_{n}$, (2) each $F_{n}$ is an $F$-space with respect to some topology $\mathscr{T}_{n}$, and (3) $\mathscr{T}$ is the $(L C)$ inductive topology determined by the continuous injections $i_{n}:\left(F_{n}, \mathscr{T}_{n}\right) \subseteq F$.

An example of such a space is ${ }_{n} \mathcal{O}_{0}$, the space of germs of holomorphic functions at 0 in $C^{n}$. Let $\left\{\omega_{k}\right\}_{k=1}^{\infty}$ be a base for the topology at 0 in $C^{n}$, then ${ }_{n} \mathcal{O}_{0}=\bigcup_{k=1}^{\infty} \mathscr{O}\left(\omega_{k}\right)$ and is the injective inductive limit of the sequence $\left\{\mathscr{O}\left(\omega_{k}\right)\right\}$.

Proposition 1.9. Let $F$ be an $L C$ space which is an injective inductive limit of $F$-spaces. If $M$ is a closed subspace of $F$, then $[E,(F / M$, quot $(F))]$ is a $C G$-pair for every $F$-space $E$.

Proof. Let $\left\{\left(F_{n}, \mathscr{T}_{n}\right)\right\}$ be the ascending sequence of $F$-spaces which determines the $F$-topology. For each $n$ we let $M_{n}$ be $M \cap F_{n}$, 
a $\mathscr{T}_{n}$-closed subspace of $F_{n}$. Hence, $F_{n} / M_{n}$ is an $F$-space, and there exists a continuous map $r_{n}: F_{n} / M_{n} \rightarrow(F / M$, quot $(F))$. Moreover, $\bigcup_{n=1}^{\infty} r_{n}\left(F_{n} / M_{n}\right)=F / M$, and the sequence $\left\{F_{n} / M_{n}\right\}$ defines an inductive topology $\mathscr{T}_{2}$ an $F / M$. We let $\mathscr{T}_{1}$ be quot $(F)$. Then $\mathscr{T}_{1} \leqq \mathscr{T}_{2}$ since each $r_{n}$ is continuous. Now $\left(F / M, \mathscr{T}_{2}\right)$ is an inductive limit of $F$-spaces, so $\left[E,\left(F / M, \mathscr{T}_{2}\right)\right]$ is a $C G$-pair for every $F$-space. Apply Lemma 1.9.

REMARK. If in Theorem 1.7 the $L C(E q)-K^{n}$ space is an injective inductive limit of $F$-spaces, then (1) $[F, F]$ is a $C G$-pair and (2) if $M$ is any subspace of $F$ which is both $F$ - and ${ }_{n} F$-closed, then $F / M$ satisfies the required hypotheses.

2. Analytic structure theorems for $F$-algebras. The results in this are concerned with continuous maps and polydises and varieties into the spectrum of an $F$-algebra in such a way that Gelfand transforms "are" holomorphic functions. We begin by fixing our notation, defining the relevant concepts and stating (mostly without proof) some results on varieties and holomorphic functions thereon.

DeFinition. An F-algebra is a separated topological algebra whose topology is determined by a countable family of submultiplicative seminorms and which is complete. A uniform F-algebra is a commutative $F$-algebra $A$ such that the Gelfand transform is a topological isomorphism onto a subalgebra of $C(M(A))$, where the latter is equipped with the topology of compact convergence. $(M(A)$ is the spectrum of $A$, the space of nonzero $C$-valued homomorphisms of $A$ equipped with the relative weak * topology.)

Definition. Let $\Omega$ be an open subset of $C$. A subset $V$ of $\Omega$ is called a subvariety of $\Omega$ if for each $z \in \Omega$ there is a neighborhood $U$ of $z$ and a family $\mathscr{F} \subseteq \mathcal{O}(U)$ (the algebra of holomorphic functions on $U$ ) such that

$$
V \cap U=\{\zeta \in U: f(\zeta)=0 \quad(f \in \mathscr{F})\} .
$$

It follows from $[6, \mathrm{p} .87]$ that the family $\mathscr{F}$ may be taken to be finite.

Definition. ${ }_{n}^{\mathscr{V}}=\left\{V \subseteq C^{n}: V\right.$ is a subvariety of a neighborhood of 0 and $0 \in V\}$.

Definition. If $V$ is a subvariety of $\Omega$ we say that $V$ is globally determined in $\Omega$ if there exists $\mathscr{F} \subseteq \mathcal{O}(\Omega)$ such that $V=\{x \in \Omega: f(z)=0$ $(f \in \mathscr{F})\}$.

We note that if $V$ is a subvariety of $\Omega$ (and is globally determined 
in $\Omega$ ), and if $\omega$ is an open subset of $\Omega$, then $V \cap \omega$ is a subvariety of $\omega$ (and is globally determined in $\omega$ ).

DeFinition. Let $V$ be a subvariety of $\Omega$, and let $f: V \rightarrow C$ be a continuous function. We say that $f$ if holomorphic on $V$ provided that for each $z \in V$ there exists a neighborhood $U$ of $z$ in $C^{n}$ and $F \in \mathcal{O}(U)$ such that $F|U \cap V=f| U \cap V$. We denote by $\mathcal{O}(V)$ the algebra of all holomorphic functions on $V$.

Proposition 2.1. Let $V$ be a globally determined subvariety of an open set $\Omega$ in $C^{n}$. We give $\mathcal{O}(V)$ the topology of uniform convergence on compact subsets of $V$. Then $\mathcal{O}(V)$ is a uniform $F$-algebra with identity. If $\Omega$ is holomorphically convex, then $\mathcal{O}(V) \cong$ $\mathscr{O}(\Omega) / \mathscr{J}(V)$, where $\mathscr{J}(V)=\{f \in \mathcal{O}(\Omega): f=0$ on $V\}$. Moreover, the isomorphism is also topological when $\mathscr{O}(\Omega) / \mathscr{J}(V)$ is given the quotient topology. Finally, in this case the spectrum of $\mathcal{O}(V)$ is $V$.

Proof. Since $\Omega$ is locally compact and $\sigma$-compact and $V$ is closed in $\Omega$, all we need to do to prove that $\mathcal{O}(V)$ is an $F$-algebra is show that $O(V)$ is complete in this topology. This is Theorem III, 7 of [12, p. 61].

Now assume that $\Omega$ is holomorphically convex. Since $\mathcal{O}(\Omega)$ is an $F$-algebra and $\mathscr{J}(V)$ is a closed ideal, we have that $\mathscr{O}(\Omega) / \mathscr{J}(V)$ with the quotient topology is an $F$-algebra and its spectrum is $V$ (see [2, p. 453]). We have maps $\mathscr{O}(\Omega) \rightarrow \mathscr{O}(V)$ (restriction) and $\mathscr{O}(\Omega) \rightarrow$ $\mathscr{O}(\Omega) / \mathscr{J}(V)$, each of which has kernel $\mathscr{J}(V)$. The latter map is clearly surjective and that the former is surjective is an immediate consequence of Cartan's Theorem B (see [6, p. 245]). Thus, the Gelfand transform maps $\mathscr{O}(\Omega) / \mathscr{F}(V)$ onto $\mathscr{O}(V)$ (if we identify $M(\mathcal{O}(\Omega) / \mathscr{I}(V)$ )) and $V$, and is injective. Now each of $\mathscr{O}(\Omega) / \mathscr{I}(V)$ and $\mathscr{O}(V)$ is an $F$-space. Thus, the Gelfand transform is a topological isomorphism.

DEFINITION. Let $V \in{ }_{n} \mathscr{Y}$ and define a relation $\sim$ on $\bigcup\{\mathcal{O}(V \cap \omega): \omega$ is a neighborhood of 0$\}$ by $f \sim g$ if, and only if, there exists a neighborhood $\omega$ of 0 such that $V \cap \omega \subseteq \operatorname{Dom}(f) \cap \operatorname{Dom}(g)$ and $f|V \cap \omega=g| V \cap \omega$. Let ${ }_{V} \mathcal{O}_{0}$ be the algebra of equivalence classes of $\mathrm{U} \mathscr{O}(V \cap \omega)$ modulo this relation with the obvious operation; i.e., ${ }_{v} \mathcal{O}_{0}=\lim _{\rightarrow 0 \in \omega} \mathcal{O}(V \cap \omega)$.

Proposition 2.2. Let $V \in_{n} \mathscr{Y}$. There exists a sequence $\left\{\omega_{k}\right\}_{k=1}^{\infty}$ of open neighborhoods of 0 such that

(1) $\omega_{1} \supseteqq \omega_{2} \supseteqq \cdots \cdot$

(2) $\left\{\omega_{k}\right\}_{k=1}^{\infty}$ is a base at 0 .

(3) $V \cap \omega_{k}$ is globally determined in $\omega_{k}(k \geqq 1)$. 
(4) if $f \in \mathcal{O}\left(V \cap \omega_{k}\right)$ and $f \mid V \cap \omega_{l}=0$ for some $l>k$, then $f=0$ on $V \cap \omega_{k}$.

(5) for each $k \in N$ the natural $\operatorname{map}_{1} i_{k}: \mathcal{O}\left(V \cap \omega_{k}\right) \rightarrow{ }_{V} \mathcal{O}_{0}$ is injective.

Proof. Fix a basis $\left\{U_{k}\right\}_{k=1}^{\infty}$ at 0 . Choose a neighborhood $\Omega$ of 0 such that $V \cap \Omega$ is globally determined in $\Omega$. Then $U_{1} \cap \Omega$ is a neighborhood of 0 and Lemma 1.8 of [4, p. 14] yields the existence of a neighborhood $\omega_{1}$ of 0 such that $\omega_{1} \subseteq U_{1} \cap \Omega$ and such that if $f \in \mathcal{O}\left(V \cap \omega_{1}\right)$ and if $f=0$ in some neighborhood of 0 , then $f=0$ in $V \cap \omega_{1}$. Now $U_{2} \cap \omega_{1}$ is a neighborhood of 0 . Continue inductively to apply Clayton's Lemma 1.8. The resulting sequence $\left\{\omega_{k}\right\}_{k=1}^{\infty}$ has the desired properties.

Definition. If $V \in_{n} \mathscr{Y}$ and $\left\{\omega_{k}\right\}_{k=1}^{\infty}$ satisfies the conclusions of Proposition 2.2 we shall call $\left\{\omega_{k}\right\}_{k=1}^{\infty}$ a $V$-basis at 0 .

We need an alternate description of ${ }_{V} O_{0}$.

Definition. ${ }_{n} \mathcal{O}_{0}$ is the algebra of germs (at 0 ) of functions holomorphic near 0 in $C^{n}$. If $V \in_{n} \mathscr{V}$, we define id $V=\left\{\alpha \in_{n} \mathcal{O}_{0}\right.$ : there exists a representative $f$ of $\alpha$ with domain $\omega$ such that $V \cap \omega \subseteq$ $\{z \in \omega: f(z)=0\}\}$.

Proposition 2.3. If $V \in_{n} \mathscr{V}$; then id $V$ is an ideal in ${ }_{n} \mathscr{O}_{0}$ which is closed with respect to both the inductive and ${ }_{n} F$-topologies on ${ }_{n} \mathcal{O}_{0}$. Moreover, ${ }_{n} \mathcal{O}_{0} / \mathrm{id} V$ is algebraically isomorphic to ${ }_{{ }^{O}}{ }_{0}$, and if we identify them, then for each open $\omega$ containing 0 the natural map of $\mathcal{O}(V \cap \omega)$ into $\left({ }_{V} \mathcal{O}_{0}\right.$, quot $\left.\left({ }_{n} \mathcal{O}_{0}\right)\right)$ is continuous.

Proof. It is clear that id $V$ is an ideal in ${ }_{n} \mathcal{O}_{0}$. That id $V$ is ${ }_{n} F$-closed is a consequence of the version of the "closure of modules theorem" found in Hörmander's book [7, p. 152]. Since the ${ }_{n} F$-topology is dominated by the ${ }_{n} \mathscr{O}_{0}$-topology (inductive topology) id $V$ must also be closed in this finer topology. We now show that ${ }_{n} \mathcal{O}_{0} / \mathrm{id} V$ and ${ }_{V} \mathcal{O}_{0}$ are algebraically the same. Define $\psi:{ }_{n} \mathcal{O}_{0} \rightarrow{ }_{V} \mathcal{O}_{0}$ by $\psi\left((f, \omega)_{0}\right) \equiv$ $(f \mid V \cap \omega, V \cap \omega)_{0}$, where we denote by $(f, \omega)_{0}$ the germ of $f \in \mathcal{O}(\omega)$, etc. It is clear that $\psi$ is a well-defined homomorphism. If $\psi\left((f, \omega)_{0}\right)=0$, then $(f \mid V \cap \omega, V \cap \omega)_{0}=0$ in ${ }_{V} \mathcal{O}_{0}$ and there exists a neighborhood $\omega^{\prime} \leqq \omega$ of 0 such that $f \mid V \cap \omega^{\prime} \equiv 0$. But then $\left(f \mid \omega^{\prime}, \omega^{\prime}\right)$ is a representative of $(f, \omega)_{0}$ and $V \cap \omega^{\prime} \leqq\left\{z \in \omega^{\prime}: f(z)=0\right\}$. Hence, $(f, \omega)_{0} \in \mathrm{id} V=$ $\operatorname{Ker} \pi$, where $\pi$ is the natural map of ${ }_{n} \mathcal{O}_{0}$ onto ${ }_{n} \mathcal{O}_{0} / \mathrm{id} V$. Clearly, $\operatorname{Ker} \pi=\mathrm{id} V \subseteq \operatorname{Ker} \psi$. That $\psi$ is surjective is a direct consequence of the definition of ${ }_{V} \mathcal{O}_{0}$. Thus $\pi$ and $\psi$ are each surjective and they 
have the same kernel. Hence, ${ }_{n} \mathcal{O}_{0} /$ id $V$ and ${ }_{V} \mathcal{O}_{0}$ are isomorphic.

Fix a neighborhood $\omega$ of 0 . If $\omega$ is holomorphically convex then $V \cap \omega$ is globally determined in $\omega$, and $\mathscr{O}(V \cap \omega)=\mathscr{O}(\omega) / \mathscr{I}(V \cap \omega)$ (and the given topology (compact convergence) is the quotient topology). We have the following diagram.

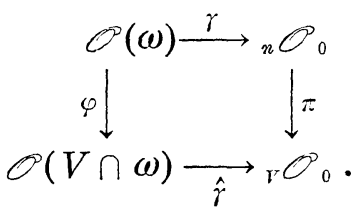

We know that $\varphi, \gamma$, and $\pi$ are continuous, when ${ }_{V} \mathcal{O}_{0}$ is given the quotient topology (carried from ${ }_{n} \mathscr{O}_{0} / \mathrm{id} V$ by the isomorphism produced above). Hence, the induced map $\hat{\gamma}$ is continuous. But $\hat{\gamma}$ is just the natural map of $\mathcal{O}(V \cap \omega)$ into ${ }_{V} \mathcal{O}_{0}$.

If $\omega$ is an arbitrary neighborhood of 0 we choose $\omega^{\prime} \leqq \omega$, a holomorphically convex neighborhood such that $V \cap \omega^{\prime}$ is globally determined in $\omega^{\prime}$. Then we have

$$
\mathscr{O}(V \cap \omega) \longrightarrow \mathcal{O}\left(V \cap \omega^{\prime}\right) \longrightarrow{ }_{V} \mathcal{O}_{0},
$$

where the first map is just a (continuous) restriction map and the second is continuous (by our argument above). The composition is the natural map of $\mathcal{O}(V \cap \omega)$ into ${ }_{V} \mathcal{O}_{0}$.

Definition. Fix $V \in_{n} \mathscr{Y}$. We say that $V$ is irreducible at 0 provided that whenever $\omega$ is a neighborhood of 0 and $V_{1}, V_{2}$ are elements of ${ }_{n} \mathscr{V}$ such that $V \cap \omega=\left(V_{1} \cap \omega\right) \cup\left(V_{2} \cap \omega\right)$, then there exists a neighborhood $\omega^{\prime}$ of 0 such that either $V \cap \omega^{\prime}=V_{1} \cap \omega^{\prime}$ or $V \cap \omega^{\prime}=V_{2} \cap \omega^{\prime}$.

Proposition 2.4. If $V \epsilon_{n} \mathscr{Y}$, then there exists a neighborhood $\omega$ of 0 and $V_{1}, \cdots, V_{m} \in{ }_{n} \mathscr{Y}$ such that

(1) each $V_{j}$ is irreducible at 0 .

(2) $\quad V \cap \omega=\bigcup_{j=1}^{m}\left(V_{j} \cap \omega\right)$.

Proof. See [6, pp. 89-90]. We shall call $\left\{V_{1}, \cdots, V_{m}\right\}$ an irreducible family for $V$.

REMARK. In [6] a stronger result is proved using germs of varieties. We shall not need the strengthened conclusion.

Proposition 2.5. If $V, V_{1}, \cdots, V_{m} \in_{n} \mathscr{Y}$ and if 


$$
V \cap \omega=\bigcup_{j=1}^{m}\left(V_{j} \cap \omega\right)
$$

for some neighborhood $\omega$ of 0 , then id $V=\bigcap_{j=1}^{m}$ id $V_{j}$ in ${ }_{n} \mathscr{O}_{0}$.

Proof. This is an easy "representative chase."

Proposition 2.6. $V \in_{n} \mathscr{Y}$ is irreducible at 0 if, and only if, id $V$ is a prime ideal in ${ }_{n} \mathcal{O}_{0}$.

Proof. See [6, p. 89].

Proposition 2.7. Let $V \in_{n}{ }_{n}$ a and let $\left\{V_{1}, \cdots, V_{m}\right\}$ be an irreducible family for $V$. Then there exists a family $\left\{\rho_{j}:{ }_{V} \mathcal{O}_{0} \rightarrow{ }_{V j} \mathcal{O}_{j}\right\}$ of quot $\left.{ }_{n} \mathcal{O}_{0}\right)$-continuous homomorphisms such that

(1) $\bigcap_{j=1}^{m} \operatorname{Ker} \rho_{j}=\{0\}$, and

(2) the following diagrams commute $(j=1, \cdots, m)$ :

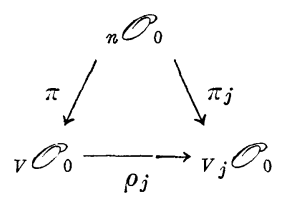

where $\pi$ and $\pi_{i}$ are the natural maps determined by the ideals id $V$ and $\operatorname{id} V_{j}$, respectively.

Proof. Since for each $j \in\{1, \cdots, m\}$ there is a unique homomorphism $\rho_{j}$ which makes the appropriate diagram commutative and since each of these induced maps is continuous, we need only verify (1). Suppose $\pi \alpha \in \bigcap_{j=1}^{m} \operatorname{Ker} \rho_{j}$ for some $\alpha \in{ }_{n} \mathcal{O}_{0}$. Then for each $j, \rho_{j} \pi(\alpha)=0$. But $\rho_{j} \pi(\alpha)=\pi_{j}(\alpha)$. Hence, $\alpha \in \bigcap_{j=1}^{m} \operatorname{Ker} \pi_{j}=\bigcap_{j=1}^{m}$ id $V_{j}=\operatorname{id} V=\operatorname{Ker} \pi$. Thus, we see that $\pi \alpha=0$.

LEMma 2.8. We define $\eta:{ }_{n} \mathcal{O}_{0} \rightarrow C$ to be the evaluation map $\eta:(f, \omega)_{0} \rightarrow f(0)$ for $(f, \omega)_{0} \in{ }_{n} \mathcal{O}_{0}$. The map $e:{ }_{V} \mathcal{O}_{0} \rightarrow C$ induced by $\eta$ and $\pi:{ }_{n} \mathcal{O}_{0} \rightarrow{ }_{n} \mathcal{O}_{0} / \mathrm{id} V \cong{ }_{V} \mathcal{O}_{0}$ is a continuous homomorphism of ${ }_{V} \mathcal{O}_{0}$ for every $V \in{ }_{n} \mathscr{Y} . \quad\left({ }_{V} \mathcal{O}_{0}\right.$ has the quot $\left({ }_{n} \mathcal{O}_{0}\right)$-topology.) (We shall call $e$ the evaluation map for ${ }_{V} \mathcal{O}_{0}$.)

Proof. The map $\eta$ is a continuous homomorphism of ${ }_{n} O_{0}$ (with respect to the ${ }_{n} \mathcal{O}_{0}$-topology) whose kernel is the maximal ideal of ${ }_{n} \mathcal{O}_{0}$. Thus, $\operatorname{Ker} \pi \subseteq \operatorname{Ker} \eta$ and the map $e$ is well-defined continuous homomorphism of ${ }_{n} \mathcal{O}_{0} /$ id $V$ to $C$.

Lemma 2.9. Let $V \in_{n}$ Y and let $\left\{V_{1}, \cdots, V_{m}\right\} \subseteq_{n} \mathscr{Y}$ be an irre- 
ducible family for $V$. For each $j \in\{1, \cdots, m\}$ we have $e_{j} \circ \rho_{j}=e$. In particular, if $\gamma \in \in_{V} \mathcal{O}_{0}$ and if $\rho_{j}(\gamma) \in C \cdot(1)_{V_{j}}$, then $\rho_{j}(\gamma)=e(\gamma) \cdot(1)_{V_{j}} \cdot$

Proof. We have the following commutative diagram

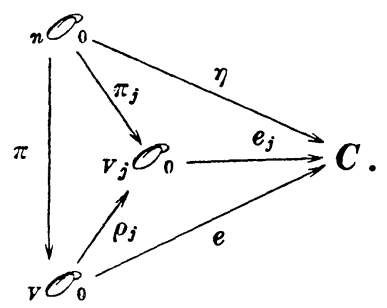

The second part of the conclusion follows from the first part.

Proposition 2.10. Let $V \in_{n} \mathscr{Y}$ and let $\left\{V_{1}, \cdots, V_{m}\right\}$ be an irreducible family for $V$. If $\gamma \in{ }_{V} \mathcal{O}_{0} \mid \boldsymbol{C} \cdot(1)_{V}$, then there exists $j \in\{1, \cdots, m\}$ such that $\rho_{j}(\gamma) \in_{V_{j}} \mathcal{O}_{0} \backslash \boldsymbol{C} \cdot(1)_{V_{j}}$.

Proof. If $\gamma \in{ }_{V} \mathcal{O}_{0}$ and if for each $j \in\{1, \cdots, m\} \rho_{j}(\gamma) \in C \cdot(1)_{V_{j}}$, then $\rho_{j}(\gamma)=e(\gamma) \cdot(1)_{V_{j}} \cdot$ Consider the element $\gamma-e(\gamma) \cdot(1)_{V}$ of ${ }_{V} \mathscr{O}_{0}$. It is clear that it belongs to $\bigcap_{\jmath=1}^{n} \operatorname{Ker} \rho_{j}(=\{0\})$. Hence, $\gamma=e(\gamma)(1)_{V}$.

We now have all the requisite information about the algebras ${ }_{V} \mathcal{O}_{0}$ to allow us to proceed with our applications.

We note that we require a homomorphism from one algebra with identity to another to map the identity of the first onto the identity of the second.

We first extend Loy's results on $F$-algebras which admit maps into ${ }_{n} \mathcal{O}_{0}$ (see $\left.[10]\right)$.

If $\beta \in{ }_{n} \mathcal{O}_{0} \mid C(1)_{0}$, then the map $T:{ }_{n} \mathcal{O}_{0} \rightarrow{ }_{n} \mathcal{O}_{0}$ defined by $T(\alpha)=\beta \alpha$ has empty point spectrum since ${ }_{n} \mathcal{O}_{0}$ is an integral domain. Also, ${ }_{n} \mathcal{O}_{0}$ is an injective inductive limit of $F$-spaces and is an $L C\left(E_{q}\right)-K^{n}$ space (see [10]); hence, satisfies the hypotheses required of the " $F$ " of Theorem 1.6.

THeOREM 2.11. Let $A$ be a commutative $F$-algebra with identity. If there exists a homomorphism $\varphi: A \rightarrow{ }_{n} \mathcal{O}_{0}$ such that rank $\varphi>1$, then there exists a neighborhood $\omega$ of 0 in $\boldsymbol{C}^{n}$ and a continuous map $\tau: \omega \rightarrow M(A)$ such that if $a \in A$, then $\hat{a} \circ \tau \in \mathcal{O}(\omega)$ and $(\hat{a} \circ \tau)_{0}=\varphi(\alpha)$.

Proof. Choose $x \in A$ such that $\varphi(x) \notin C \cdot(1)_{0}$. Define $S: A \rightarrow A$ and $T:{ }_{n} \mathcal{O}_{0} \rightarrow{ }_{n} \mathcal{O}_{0}$ by $S(\alpha)=x \alpha(\alpha \in A)$ and $T(\alpha)=\varphi(x) \alpha\left(\alpha \in{ }_{n} \mathcal{O}_{0}\right)$. It is clear that (1) $S$ is a continuous linear map, (2) $T$ is a lower triangular operator with empty point spectrum, and (3) $\varphi S=T \varphi$. In the light 
of our comments preceding the statement of this theorem we can apply Theorem 1.6 and conclude that $\varphi$ is continuous. Let $\left\{\omega_{k}\right\}_{k=1}^{\infty}$ be a base at 0 in $C^{n}$ consisting of holomorphically convex open sets and let $i_{k}: \mathcal{O}\left(\omega_{k}\right) \rightarrow{ }_{n} \mathcal{O}_{0}$ be the natural injection for each $k \in N$. We apply Lemma 1.5. There exists $l \in N$ such that $\varphi(A) \subseteq i_{l}\left(\mathcal{O}\left(\omega_{l}\right)\right)$ and $i_{l}^{-1} \circ \varphi$ is a continuous homomorphism of $A$ into $\mathscr{O}\left(\omega_{l}\right)$. Call this map $\psi$, let $\omega=\omega_{\iota}$ and let $\tau: \omega \rightarrow M(A)$ be the dual map of $\psi$ restricted to $\omega=M(\mathcal{O}(\omega)) \subseteq \mathscr{O}(\omega)^{*}$. We have used here the fact that $M(\mathcal{O}(\omega))=\omega$, a direct corollary of Theorem 7.2 .10 of Hörmander's book ([7, p. 171]) which implies that $\omega \ni \zeta \rightarrow$ (evaluation at $\zeta) \in M(\mathcal{O}(\omega))$ is surjective. That this map is topological follows from the fact that the weak topology on $\omega$ determined by $\mathcal{O}(\omega)$ is exactly the $C^{n}$ topology on $\omega$. If $\zeta \in \omega$, then $\tau(\zeta) \in M(A)$ is defined by $\tau(\zeta): a \rightarrow \psi(a)(\zeta)$. Hence, $(\hat{a} \circ \tau)(\zeta)=\tau(\zeta)(a)=\psi(a)(\zeta)$ and $\hat{a} \circ \tau=\psi(a) \in \mathcal{O}(\omega)$. Also, $(a \circ \tau)_{0}=$ $(\psi(a))_{0}=i_{l}(\psi(a))=\phi(a)$.

Let $W \in_{n} \mathscr{Y}$ be irreducible at 0 . Suppose $\beta \in{ }_{n} \mathcal{O}_{0}$ has the property that $\pi \beta \in{ }_{W} \mathcal{O}_{0} \mid C(1)_{W}$. Then the map $T:{ }_{n} \mathcal{O}_{0} \rightarrow{ }_{n} \mathcal{O}_{0}$ defined by $T(\alpha)=$ $\beta \alpha\left(\alpha \in{ }_{n} \mathcal{O}_{0}\right)$ leaves id $W$ invariant; hence, induces $\hat{T}:{ }_{W} \mathcal{O}_{0} \rightarrow{ }_{W} \mathcal{O}_{0}$ and since $i d W$ is a prime ideal, the algebra ${ }_{W} \mathcal{O}_{0}$ is an integral domain and $\hat{T}$ has empty point spectrum ( $\hat{T}$ is multiplication by $\pi \beta)$. Also, ${ }_{w} \mathcal{O}_{0}$ satisfies the conclusions of Proposition 1.9. Thus, there is no obstruction to our applying Theorem 1.7 to a situation like the one we have described here.

THEOREM 2.12. Let $A$ be a commutative F-algebra with identity. Suppose $V \in_{n} \mathscr{Y}$ and there exists a homomorphism $\varphi: A \rightarrow{ }_{V} \mathcal{O}_{0}$ such that rank $\varphi>1$. Then there exists a neighborhood $\omega$ of 0 in $C^{n}$ and $a$ continuous map $\tau: V \cap \omega \rightarrow M(A)$ such that if $a \in A$, then $\hat{a} \circ \tau \in \mathcal{O}(V \cap \omega)$ and $(\hat{a} \circ \tau)_{0}=\varphi(a)$.

Proof. Fix an irreducible family $\left\{V_{1}, \cdots, V_{m}\right\} \subseteq{ }_{n} \mathscr{V}$ for $V$. Let $E=\left\{j \in\{1, \cdots, m\}: \rho_{j} \varphi\right.$ has rank $\left.>1\right\}$. We note that Proposition 2.10 and our hypothesis regarding the rank of $\varphi$ imply that $E$ is nonempty. Fix $j \in E$. We shall show that $\rho_{j} \varphi$ is continuous. Choose $x \in A$ such that $\rho_{j} \varphi(x) \notin C \cdot(1)_{V_{j}}$, and choose $\beta \in{ }_{n} \mathcal{O}_{0}$ such that $\pi_{j} \beta=\rho_{j} \varphi(x)$. We define $S: A \rightarrow A$ and $T:{ }_{n} O_{0} \rightarrow{ }_{n} \mathcal{O}_{0}$ by $S(\alpha)=x a(\alpha \in A)$ and $T(\alpha)=$ $\beta \alpha\left(\alpha \in{ }_{n} \mathcal{O}_{0}\right)$. Clearly (1) $S$ is a continuous linear map, (2) $T$ is a lower triangular operator on ${ }_{n} \mathcal{O}_{0}$ and (3) $\rho_{j} \varphi S=\hat{T}_{j} \rho_{j} \varphi\left(\rho_{j} \varphi S(a)=\rho_{j}(\varphi(x a))=\right.$ $\left.\rho_{j}(\varphi(x) \varphi(a))=\rho_{j} \varphi(x) \rho_{j} \varphi(a)=\pi_{j} \beta \rho_{j} \varphi(a)=\widehat{T}_{j}\left(\rho_{j} \varphi(a)\right)\right)$. Since ${ }_{V_{j}} \mathcal{O}_{0}$ is an integral domain and $\pi_{j} \beta \notin \boldsymbol{C} \cdot(1)_{V_{j}}$ we conclude that $\hat{T}_{j}$ (which is just multiplication by $\pi_{j} \beta$ ) has empty point spectrum. Theorem 1.7 implies that $\rho_{j} \varphi$ is continuous. We select one $k \in E$.

Let $j \in\{1, \cdots, m\} \backslash E$. We shall show that $\rho_{j} \rho$ is continuous. Since $j \notin E$ we have $\rho_{j} \varphi(A) \subseteq C(1)_{V_{j}}$; hence, for each $a \in A$ we must 
have $\rho_{j}(\varphi(a))=e(\varphi(a)) \cdot(1)_{V_{j}}$ by Lemma 2.9. But $e(\varphi(a))=e_{k}\left(\rho_{k} \varphi(a)\right)$ (also by Lemma 2.9). Hence, $\rho_{j} \circ \varphi(a)=e_{k} \circ \rho_{k} \varphi(a) \cdot(1)_{V_{j}}$. But $e_{k}$ and $\rho_{k} \rho$ are continuous maps ( $e_{k}$ is continuous by Lemma 2.8 and $\rho_{k} \rho$ is continuous by the first part of this proof). Thus, $\rho_{j} \Phi$ is continuous. Since $\bigcap_{j=1}^{m} \operatorname{Ker} \rho_{j}=\{0\}$ (by Proposition 2.7) we can apply Lemma 1.3 to conclude that $\varphi$ is continuous.

We now fix a $V$-basis $\left\{\omega_{k}\right\}_{k=1}^{\infty}$ at 0 (see the Definition following Proposition 2.2), and for each $i_{k}$ we let $i_{k}: \mathcal{O}\left(V \cap \omega_{k}\right) \rightarrow{ }_{V} \mathcal{O}_{0}$ be the natural (continuous, by Proposition 2.3) injection of $\mathscr{O}\left(V \cap \omega_{k}\right)$ into ${ }_{v} \mathcal{O}_{0}$ (with the quot $\left({ }_{n} \mathcal{O}_{0}\right)$-topology). Since $A$ and the spaces $\mathcal{O}\left(V \cap \omega_{k}\right)$ are all $F$-spaces and the maps $\varphi, i_{k l}(k \in N)$ are all continuous and since $\left({ }_{V} \mathcal{O}_{0}\right.$, quot $\left.\left({ }_{n} \mathcal{O}_{0}\right)\right)$ is separated we can apply Lemma 1.5. Thus, there exists $l \in N$ such that $\varphi(A) \subseteq i_{l}\left(\mathcal{O}\left(V \cap \omega_{l}\right)\right)$ and $i_{l}^{-1} \circ \varphi: A \rightarrow \mathcal{O}\left(V \cap \omega_{l}\right)$ is continuous. Choose a holomorphically convex open neighborhood $\omega$ of 0 such that $\omega \subseteq \omega_{l}$, noting that $V \cap \omega$ is globally determined in $\omega$, and let $j: \mathscr{O}\left(V \cap \omega_{l}\right) \rightarrow \mathscr{O}(V \cap \omega)$ be the (continuous) restriction map. We set $\psi=j \circ i_{j}^{=1} \circ \varphi: A \rightarrow \mathscr{O}(V \cap \omega)$ and note that $\psi$ is a continuous homomorphism of $F$-algebras with identity. We let $\tau$ be the restriction of the dual map of $\psi$ to $V \cap \omega=M(\mathcal{O}(V \cap \omega))$. The other conclusions of the theorem are obtained exactly as in the proof of Theorem 2.11.

3. Analytic structure theorems for natural systems. In this section we apply Theorems 2.11 and 2.12 in order to extend these results to natural systems.

Definition. A natural system is a pair $[X, A]$ such that

(1) $X$ is a Hausdorff space,

(2) $A$ is an algebra of continuous functions on $X$ containing the constant functions.

(3) The weak topology on $X$ generated by $A$ is the given topology on $X$, and

(4) if $\varphi: A \rightarrow C$ is a $k$-continuous homomorphism, then there exists $x \in X$ such that $\varphi(f)=f(x)$ for each $f \in A$, where " $k$ " denotes the topology of compact convergence on $A$ (inherited from $C(X)$ ).

Note. We shall call $X$ the spectrum of the natural system $[X, A]$.

Definition. A natural system $[X, A]$ satisfies $(*)$ provided that (1) $X$ is locally compact, and (2) every open subset of $X$ is $\sigma$-compact.

Definition. Let $[X, A]$ be a natural system, and let $K$ be a compact subset of $X$. The A-convex hull of $K$ is the set $\operatorname{hull}_{A}(K)=$ $\left\{x \in X:|f(x)| \leqq\|f\|_{K}\right.$ for every $\left.f \in A\right\}$, where $\|\cdot\|_{K}$ is the supremum- 
on- $K$ seminorm on $A$. A subset $E$ of $X$ is called $A$-convex provided that $\operatorname{hull}_{A}(K) \subseteq E$ whenever $K$ is a compact subset of $E$.

Proposition 3.1. Let $[X, A]$ be a natural system. An open subset $U$ of $X$ is $A$-convex if, and only if, $[U, A \mid U]$ (equivalently, $\left[U, \overline{A \mid U^{k}}\right]$ ) is a natural system.

\section{Proof. This is just Proposition 1.3 of [13].}

Proposition 3.2. Let $[X, A]$ be a natural system. If $p \in X$, then there exists a base for the topology at $p$ which consists of A-convex open sets.

Proof. Fix $p \in X$ and let $U$ be a neighborhood of $p$. Since the topology of $X$ is the $A$-topology there exist $f_{1}, \cdots, f_{m} \in A$ such that each $f_{j}(p)=0$ and $V\left(f_{1}, \cdots, f_{m}\right)=\left\{x \in X:\left|f_{j}(x)\right|<1, j=1, \cdots, m\right\} \subseteq U$. It is easily verified that $V\left(f_{1}, \cdots, f_{m}\right)$ is $A$-convex.

Let $[X, A]$ be a natural system with $X$ a locally compact space. For each open subset $U$ of $X$ we set $A_{U}={\overline{A \mid U^{k}}}^{k}$, the $k$-completion of $A \mid U$. Since $X$ is locally compact, so is $U$. Hence, $\overline{A \mid U^{k}}$ is just the closure of $A \mid U$ in $C(U)$. If $U$ and $V$ are open and $U \subseteq V$, we define $r_{V}^{U}: A_{V} \rightarrow A_{U}$ by $r_{V}^{U}(f)=f \mid U$ for $f \in A_{V}$. It is easily verified that each $r_{V}^{V}$ is a continuous homomorphism of algebras. Moreover, $\left\{\left(A_{U} ; U\right.\right.$ open $),\left(r_{V}^{U} ; U, V\right.$ open, $\left.\left.U \subseteq V\right)\right\}$ is a presheaf on $X$. We denote by $\mathscr{A}$ the sheaf generated by this presheaf and by $\mathscr{A}_{p}$ the stalk of $\mathscr{A}$ at $p$. We make some observations about $\mathscr{A}_{p}$. First, $\mathscr{A}_{p}=$ $\lim _{\rightarrow}\left\{\left(A_{U} ; p \in U\right.\right.$ (open) $),\left(r_{U}^{V} ; U, V\right.$ open, $\left.\left.p \in U \subseteq V\right)\right\}$, and $\mathscr{A}_{p}$ is a local algebra whose maximal ideal consists of all $\alpha \in \mathscr{C}_{p}$ such that there exists an open set $U$ containing $p$ and $f \in A_{U}$ for which $f(p)=0$ and $r_{U}(f)=\alpha$. We note also that $\mathscr{A}$ is a subsheaf of $\mathscr{C}$ (the sheaf of germs of continuous functions on $X$ ) and obtain another way of regarding $\mathscr{A}_{p}$ : the germ of $f \in C(U)$ at $p$ belongs to $\mathscr{A}_{p}$ if, and only if, there exists a neighborhood $V$ of $p$ such that $V \subseteq U$ and $f \mid V \in A_{V}$. We shall use the "germ" notation, writing " $(f)_{p}$ " for " $r_{U}(f)$ " whenever $f \in A_{U}$ ( $r_{U}$ being the natural map of $A_{U}$ into $\left.\mathscr{A}_{p}\right)$, and " $f \mid V$ " instead of " $r_{U}^{V}(f)$ " $\left(f \in A_{U}, V \subseteq U\right)$. We refer the reader to Chapter IV of [6] for material on sheaves and presheaves.

Theorem 3.3. Let $[X, A]$ be a natural system which satisfies (*). Suppose $p \in X$ and there exists a homomorphism $\varphi: \mathscr{A}_{p} \rightarrow{ }_{n} \mathcal{O}_{0}$ having rank $>1$. Then there exists an A-convex open set $U$ in $X$ containing $p$, a polydisc $\Delta$ about 0 in $C^{n}$, and a continuous map $\tau: \Delta \rightarrow U$ such that 
(1) $\tau(0)=p$,

(2) if $f \in A_{U}$, then $f \circ \tau \in \mathcal{O}(\Delta)$, and

(3) if $f \in A_{U}$, then $(f \circ \tau)_{0}=\varphi\left((f)_{p}\right)$.

Proof. Fix a base $\mathscr{C}_{p}=\{U\}$ for the topology at $p$ consisting of $A$-convex open sets. If $\varphi\left(\mathscr{A}_{p}\right)$ has dimension $>1$, then there exists $U \in \mathscr{U}_{p}$ such that $\varphi_{\circ} r_{U}\left(A_{U}\right)$ has dimension $>1$. Since $U$ is $\sigma$-compact, $A_{U}$ is an $F$-algebra. We apply Theorem 2.11 and obtain a polydisc $\Delta$ about 0 in $C^{n}$ and a continuous map $\tau: \Delta \rightarrow M\left(A_{U}\right)=U$ (by Proposition 3.1), such that $f \circ \tau \in \mathcal{O}(\Delta)$ and $(f \circ \tau)_{0}=\varphi r_{V}(f)=\varphi\left((f)_{p}\right)$. We know that $\tau(0)=q \in U$. If $q \neq p$, then we can choose $f \in A_{U}$ such that $f(g)=0, f(p) \neq 0$. Since $f(p) \neq 0$ we have that $(f)_{p}$ has an inverse in $\mathscr{A}_{p}$. Hence, $\varphi\left((f)_{p}\right)$ is invertible in ${ }_{n} \mathcal{O}_{0}$. But $\varphi\left((f)_{p}\right)=(f \circ \tau)_{0}$ and $f \circ \tau(0)=0$ since $\tau(0)=q$ and $f(q)=0$. Thus, we must have $q=p$.

Theorem 3.4. Let $[X, A]$ be a natural system which satisfies (*). Suppose $p \in X$ and there exist $V \in{ }_{n} \mathscr{Y}$ and a homomorphism $\varphi: \mathscr{A}_{p} \rightarrow{ }_{V} \mathcal{O}_{0}$ having rank $>1$. Then there exists an A-convex open neighborhood $U$ of $p$ in $X$, a holomorphically convex open neighborhood $\omega$ of 0 in $\boldsymbol{C}^{n}$ and a continuous map $\tau: V \cap \omega \rightarrow U$ such that

(1) $\tau(0)=p$,

(2) if $f \in A_{U}$, then $f \circ \tau \in \mathcal{O}(V \cap \omega)$, and

(3) if $f \in A_{U}$, then $(f \circ \tau)_{0}=\varphi\left((f)_{p}\right)$.

Proof. The proof is essentially the same as that of the preceding theorem: Theorem 2.12 rather than 2.11 is invoked at the appropriate place.

LEMma 3.5. Let $[X, A]$ be a natural system with $X$ locally compact and let $U$ be an A-convex open subset of $X$. If $h_{1}, \cdots, h_{n} \in A_{U}$ and if we set $h=\left(h_{1}, \cdots, h_{n}\right): U \rightarrow C^{n}$, then $h^{-1}(\Delta)$ is an A-convex open set for each polydisc $\Delta$ in $\boldsymbol{C}^{n}$.

Proof. Fix a compact subset $K$ of $h^{-1}(\Delta)$. If $x \in$ hull $_{A_{U}}(K)$, then for each polynomial $p$ we have $|p h(x)| \leqq\|p h\|_{K}=\|p\|_{h(K)}$ and conclude that $h(x) \in$ hull $_{\text {Poly }}(h(K)) \subseteq \Delta$. Thus, $x \in h^{-1}(\Delta)$. Now, if $x \in \operatorname{hull}_{A}(K)$, then $x \in U$ ( $U$ is $A$-convex) and if $f \in A_{U}$, then for each $\varepsilon>0$ there exists $f_{\varepsilon} \in A$ such that $\left\|f-f_{\varepsilon}\right\|_{K \cup\{x\}}<\varepsilon$. But then $|f(x)| \leqq|f(x)| \leqq$ $\left|f_{\varepsilon}(x)\right|+\varepsilon \leqq\left\|f_{\varepsilon}\right\|_{K}+\varepsilon \leqq\|f\|_{K}+2 \varepsilon$. Since $\varepsilon>0$ is arbitrary, we conclude that $x \in \operatorname{hull}_{A_{U}}(K)$. Hence, $x \in h^{-1}(\Delta)$.

We now include a sketch of an extension of Proposition 3.25 of [3a] to $F$-algebras.

LEMMA 3.6. Let $A$ be a uniform F-algebra with identity and 
assume that the spectrum $X$ of $A$ is locally compact. Let $p$ be a $G_{\delta}$-point in $X, U$ a neighborhood of $p, Y$ a topological space, and $h: U \rightarrow Y$. If for each $a \in A$ there exists a complex-valued function $g$ defined in a neighborhood of $h(p)$ such that $a=g \circ h$ in some neighborhood of $p$, then $h$ is injective in some neighborhood of $p$.

Proof. Using the local compactness of $X$ we choose a nested base $\left\{U_{j}\right\}_{j=1}^{\infty}$ at $p$ such that $U_{1} \subseteq U$. Fix an ascending sequence $\left\{K_{j}\right\}_{j=1}^{\infty}$ of compact sets such that $U \subseteq K_{1}, K_{n} \leqq K_{n+1}^{0}(n \geqq 1)$, and $\bigcup_{n=1}^{\infty} K_{n}=X$ (we may assume that $U$ is relatively compact by intersecting it with $\left.K_{1}^{0}\right)$.

If $h$ is not injective in any neighborhood of $p$, then as in [3a] we may choose sequences $\left\{x_{j}\right\}_{j=1}^{\infty}$ and $\left\{y_{j}\right\}_{j=1}^{\infty}$ in $X$ such that for each $j$ we have $x_{j} \neq y_{j}$, and (\#) for each $a \in A$ there exists $j(a)$ such that $a\left(x_{j}\right)=a\left(y_{j}\right)(j \geqq j(a))$. Construct a sequence $\left\{a_{j}\right\}_{j=1}^{\infty}$ in $A$ such that for each $j$ we have $\left\|a_{j}\right\|_{j}=1, a_{j}\left(x_{j}\right) \neq a_{j}\left(y_{j}\right)$, and $a_{j}\left(x_{k}\right)=a_{j}\left(y_{k}\right)(k>j)$. (It may be necessary to use a subsequence of the $x_{j}$ 's to accomplish this.) The estimates which finish the proof can be obtained by the same procedures used in [3a]. We state the main steps. Let $b_{1}=a_{1}$ and define $b_{j+1}=6^{-1} a_{j+1}\left[b_{j}\left(x_{j}\right)-b_{j}\left(y_{j}\right)\right]$ for $j \geqq 1$. It is easily checked that (1) $b_{j}\left(x_{j}\right) \neq b_{j}\left(y_{j}\right)$ for each $j$, (2) $\left\|b_{j}\right\|_{j} \leqq 3^{-j+1}$ for each $j$, and (3) $\left\|b_{j+k}\right\|_{j+k} \leqq 2^{-1} 3^{-k}\left|b_{j}\left(x_{j}\right)-b_{j}\left(y_{j}\right)\right|$ for each $j$ and $k$. We conclude from (2) that $\sum_{j} b_{j}$ converges in $A$ (say, to (b)), and from (3) that for each $j$ we have $\left|b\left(x_{j}\right)-b\left(y_{j}\right)\right| \geqq 2^{-1}\left|b_{j}\left(x_{j}\right)-b_{j}\left(y_{j}\right)\right|>0$, a violation of $(\#)$.

Lemma 3.7. Let $[X, A]$ be a natural system which satisfies (*). Suppose $p \in X$ and $\varphi: \mathscr{A}_{p} \rightarrow{ }_{v} \mathcal{O}_{0}$ is a surjective homomorphism. Choose an A-convex neighborhood $U$ of $p$ and $h_{1}, \cdots, h_{n}$ such $\varphi\left(\left(h_{j}\right)_{p}\right)=\left(z_{j} \mid V\right)_{0}$ $(j=1, \cdots, n)$. There exists a polydisc $\triangle$ about 0 in $C^{n}$ and a continuous map $\tau: V \cap \Delta \rightarrow U$ such that (1) $\tau(0)=p$, and (2) if $f \in A_{U}$, then $f \circ \tau \in \mathcal{O}(V \cap \Delta)$ and $(f \circ \tau)_{0}=\varphi\left((f)_{p}\right)$. (Theorem 3.4.)

Suppose $W$ is an A-convex neighborhood of $p$ which is contained in $U$. Then there exists a neighborhood $\omega$ of 0 in $C^{n}$ such that (1) $\omega \subseteq \Delta$, (2) $\varphi \circ r_{W}\left(A_{W}\right) \subseteq i_{\omega}(\mathcal{O}(V \cap \omega))$, and (3) $i_{\omega}$ is injective. Let $\psi_{W}=i_{\omega}^{-1} \circ \varphi \circ r_{W}: A_{W} \rightarrow \mathcal{O}(V \cap \omega)$, and let $\tau_{W}: V \cap \omega \rightarrow W$ be the dual of $\psi_{W}$ restricted to $V \cap \omega\left(\subseteq M_{\left.\mathcal{O}_{(V \cap \omega)}\right)}\right.$. Then (4) if $f \in A_{U}$, then $\psi_{W}(f \mid W)=\psi_{U}(f) \mid V \cap \omega$, and (5) $\tau_{W}=\tau \mid V \cap \omega$.

Proof. Statements (1), (2), and (3) follow from Theorem 3.4 and the fact that $\varphi\left(r_{W}\left(A_{W}\right)\right)$ has dimension greater than one. We next consider statement (4). Recall that we obtain $\Delta$ and $\psi_{U}: A_{U} \rightarrow$ $\mathcal{O}(V \cap \Delta)$ by first choosing a neighborhood $\omega^{\prime}$ of 0 such that $\varphi_{\circ} r_{U}\left(A_{U}\right) \subseteq$ $i_{\omega^{\prime}}\left(\mathcal{O}\left(V \cap \omega^{\prime}\right)\right)$ and $i_{\omega^{\prime}}$ is injective, with $\psi_{U}^{\prime}: A_{U} \rightarrow \mathcal{O}\left(V \cap \omega^{\prime}\right)\left(=i_{\omega^{\prime}}^{-1} \circ \varnothing_{\circ} \circ r_{U}\right)$. 
We have the following diagram (unlabelled arrows are restriction maps).

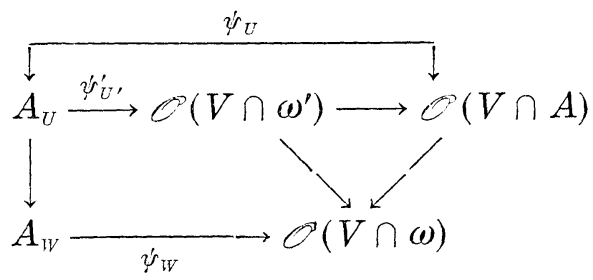

Statement (4) follows.

If $\zeta \in V \cap \omega$, then $\tau_{W}(\zeta) \in W$ satisfies $f\left(\tau_{W}(\zeta)\right)=\psi_{W} f(\zeta)\left(f \in A_{W}\right)$. In particular, for $f \in A_{U}$ we have $f\left(\tau_{W}(\zeta)\right)=\left(\psi_{U}(f) \mid V \cap \omega\right)(\zeta)=$ $\psi_{U}(f(\zeta))=f(\tau(\zeta))$. Since $A_{U} \mid W$ separates the points of $W$ we conclude that $\tau_{W}=\tau$ on $V \cap \omega$.

THEOREM 3.8. Let $[X, A]$ be a natural system which satisfies (*).

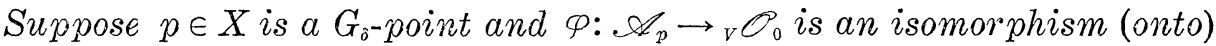
for some $V \in_{n} \mathscr{Y}$. Then there exists an $A$-convex open neighborhood $W_{0}$ of $p$, a polydisc $\Delta_{0}$ about 0 in $C^{n}$, and a homeomorphism $\tau_{0}$ of $V \cap \Delta_{0}$ onto $W_{0}$ such that (1) $\tau_{0}(0)=p$, (2) $f \rightarrow f \circ \tau_{0}$ is a topological isomorphism of $A_{W_{0}}$ onto $O\left(V \cap \Delta_{0}\right)$, and (3) if $f \in A_{W_{0}}$, then $\varphi\left((f)_{p}\right)=$ $\left(f \circ \tau_{0}\right)_{0}$.

Proof. Fix an $A$-convex open neighborhood $U$ of $p$ and $h_{1}, \cdots$, $h_{n} \in A_{U}$ such that $\varphi\left(\left(h_{j}\right)_{p}\right)=\left(z_{j} \mid V\right)_{0}(j=1, \cdots, n)$. Choose a polydisc $\Delta$ about 0 in $C^{n}$ and $\tau: V \cap \Delta \rightarrow U$ a continuous map such that $\tau(0)=p$ and $f \circ \tau \in \mathcal{O}(V \cap \Delta)$ and $(f \circ \tau)_{0}=\varphi\left((f)_{p}\right)$ for each $f \in A_{U}$. Let $\psi$ be the map $f \rightarrow f \circ \tau$ of $A_{U}$ into $\mathcal{O}(V \cap \Delta)$. Then $\psi\left(A_{U}\right)$ contains $\left\{z_{1}\left|V, \cdots, z_{n}\right| V\right\}$; hence $\tau$ is a relative homeomorphism. Let $h=\left(h_{1}, \cdots, h_{n}\right): U \rightarrow C^{n}$. The map $h$ is continuous and $h \tau$ is the identity on $V \cap \Delta$.

We now verify two facts: (1) $p \in \operatorname{int}\left[h^{-1}(V \cap A)\right]$ and (2) $h$ is injective in some neighborhood of $p$.

(1) Let $U_{0}=h^{-1}(4)$. Then, by Lemma 3.5, $U_{0}$ is an $A$-convex neighborhood of $p$. Let $\left\{U_{j}\right\}_{j=1}^{\infty}$ be a nested base at $p$ such that $U_{1} \subseteq U_{0}$, let $E=h^{-1}(V \cap \Delta)$ and define for $S \subseteq U_{0}$ the closed ideal $I(S)$ in $A_{U_{0}}$ by setting $I(S)=\left\{f \in A_{U_{0}}: f \mid S=0\right\}$. If $f \in I(E)$, then $f \mid E=0$. By Lemma 3.7 we have $\tau_{U_{0}}=\tau$ on $V \cap \omega_{0}\left(\omega_{0}\right.$ an appropriate neighborhood of 0 in $C^{n}$ corresponding to $\left.U_{0}\right)$. If $\zeta \in V \cap \omega_{0}$, then $\tau_{U_{0}}(\zeta) \in E$, since $h\left(\tau_{U_{0}}(\zeta)\right)=h(\tau(\zeta))=\zeta \in V \cap \Delta$. Thus, $f \circ \tau_{U_{0}}=0$ and $\varphi\left((f)_{p}\right)=$ $\left(f \circ \tau_{U_{0}}\right)_{0}=0$. But $\varphi$ is injective; hence, $(f)_{p}=0$ and $f \mid U_{j}=0$ for some $j$. We have shown that $I(E)=\bigcup_{j=1}^{\infty} I\left(E \cup U_{j}\right)$. Now $I(E)$ is an $F$-space and each $I\left(E \cup U_{j}\right)$ is a closed subspace of $I(E)$. Since $I(E)$ is a Baire space and proper closed subspaces must have empty interior 
we conclude that $I(E)=I\left(E \cup U_{k}\right)$ for some $k$. If there exists $x \in U_{k} \backslash E$, then $h(x) \in \Delta \backslash V \cap \Delta$ and there exists $g \in \mathcal{O}(\Delta)$ such that $g(h(x))=1$ and $g \mid V \cap \Delta=0$. Since $g \circ h \in A_{U_{0}}$ we have $I(E) \neq I\left(E \cup U_{k}\right)$. (Approximate $g$ on $\Delta$ by polynomials $\left\{p_{m}\right\}$, then $p_{m}\left(h_{1}, \cdots, h_{n}\right) \in A_{U_{0}}$ and $p_{m}\left(h_{1}, \cdots, h_{n}\right) \rightarrow$ $g \circ h$ in $\left.A_{U_{0}}\right)$.

(2) We shall apply Lemma 3.6 with $X=U, U_{0}$ the neighborhood of $p$, and $h: U_{0} \rightarrow C^{n}$ the function in question. Fix $f \in A_{U}$. Then $f \circ \tau \in \mathcal{O}(V \cap \Delta)$ and there exists $g \in \mathcal{O}(\Delta)$ such that $f \circ \tau=g \mid V \cap \Delta$. Consider $g \circ h \in A_{U_{0}}$. We claim that $\varphi\left((f)_{p}\right)=\varphi\left((g \circ h)_{p}\right)$. The latter is $\left(g \circ h \circ \tau_{U_{0}}\right)_{0}\left(\right.$ Lemma 3.7) $=\left(g \mid V \cap \omega_{0}\right)_{0}$. The former is $(f \circ \tau)_{0}$. But $(f \circ \tau)_{0}=(g \mid V \cap \Delta)_{0}=\left(g \mid V \cap \omega_{0}\right)_{0}$. Thus, $(g \circ h)_{p}=(f)_{p}$ and $f=g \circ h$ near p. Apply Lemma 3.6.

We now choose an $A$-convex open neighborhood $W$ of $p$ such that $W \sqsubseteq h^{-1}(V \cap \Delta)$ and $h$ is injective on $W$. Choose a polydisc $\Delta_{0}$ about 0 in $C^{n}$ such that $V \cap \Delta_{0} \subseteq \tau^{-1}(W)$. We set $W_{0}=h^{-1}\left(V \cap \Delta_{0}\right) \cap W$ ( $\left.=h^{-1}\left(\Delta_{0}\right) \cap W\right)$. Then $W_{0}$ is an $A$-convex open neighborhood of $p$ and (1) $V \cap \Delta_{0}=\tau^{-1}\left(W_{0}\right)$, (2) $h\left(W_{0}\right) \subseteq V \cap \Delta_{0}$, (3) $\tau h$ is the identity on $W_{0}$, and (4) $h \tau$ is the identity on $V \cap \Delta_{0}$. Statements (2) and (4) are clear. The first follows from

$$
\begin{aligned}
& \tau^{-1}\left(W_{0}\right)=\tau^{-1}\left(h^{-1}\left(V \cap \Delta_{0}\right) \cap W\right)=\tau^{-1} h^{-1}\left(V \cap \Delta_{0}\right) \cap \tau^{-1}(W) \\
& \quad=(h \tau)^{-1}\left(V \cap \Delta_{0}\right) \cap \tau^{-1}(W)=\left(V \cap \Delta_{0}\right) \cap \tau^{-1}(W)=V \cap \Delta_{0} .
\end{aligned}
$$

The third is proved as follows. If $x \in W_{0}$, then $\tau h(x) \in W_{0} \subseteq W$ and $h(\tau h(x))=h \tau(h(x))=h(x)$. But $h$ is injective on $W$, so $\tau h(x)=x$.

Let $\tau_{0}$ be the surjective homeomorphism $\tau \mid \Delta_{0} \cap V: \Delta_{0} \cap V \rightarrow W_{0}$. This map satisfies the conclusions of the theorem.

REMARKS. We close with some remarks on the preceding results.

(1) There is an obvious corollary to the special natural system $[M(A), A]$ where $A$ is a uniform $F$-algebra with identity. This corollary is in the spirit of Clayton's localization of Gleason's theorem, although we do not use the same sheaf.

(2) It is clear that the condition (*) could be replaced by a weaker condition concerning only the nature of the topology of $X$ near $p$. For example, one alternative would be $(* p)$ : there exists a base for the topology at $p$ consisting of $A$-convex, $\sigma$-compact open sets ( $X$ is assumed locally compact).

(3) We assumed in the four main theorems that the rank of the homomorphism $\varphi$ was $>1$. There are two reasons for this assumption. First, it now appears that in Theorems 2.11 and 2.12 the assumption is necessary in order to guarantee that $\varphi$ is continuous: Benedetto (see [1]) asserts the existence of commutative $F$-algebras which admit discontinuous complex-valued homomorphisms. Second, even if $\varphi$ is continuous, the conclusion is guaranteed to be meaningless: $\tau(\omega)$ (or 
$\tau(V \cap \omega)$ ) will be a single point in $M(A)$ (or in $U$ ) and $f \circ \tau$ will be a constant function for any $f$ defined on $M(A)$ (or $U$ ). Thus, some restriction on the nature of $\phi$ is essential in order to avoid the certainty of a trivial conclusion.

(4) The sheaf used by Carpenter and Loy in the case $[M(A), A]$ was constructed so that each algebra $A_{U}^{\prime}$ was an $F$-algebra (without topological restrictions on $M(A)$ ). However, there are two reasons we choose our approach rather than theirs. First, their algebras $A_{U}^{\prime}$ are not in general algebras of continuous functions on $C(U)$ (they are completions of $A \mid U$ relative to a topology with respect to which $C(U)$ is not in general complete). Second, the spectrum of $A_{U}^{\prime}$ will be unrelated to $M(A)$ unless the open set $U$ is of the type we choose to consider.

(5) The example given in [3a] to show the necessity of the assumption that $p$ be a $G_{\hat{o}}$-point remains valid in our setting.

\section{REFERENCES}

1. John J. Benedetto, The Helson-S set problem and discontinuous homomorphism on metric algebras, (preprint).

2. R. M. Brooks, The structure space of a commutative locally m-convex algebra, Pacific J. Math., 25 (1968), 443-454.

3. R. L. Carpenter, Singly generated homogeneous F-algebras, Trans. Amer. Math. Soc., 150 (1970), 457-469.

4. D. D. Clayton, A local characterization of analytic structure in a commutative Banach algebra, Symposium on Several Complex Variables, Park City, Utah, 1970, Springer-Verlag Lecture Notes 184 (1971).

5. R. E. Edwards, Functional Analysis, Theory and Applications, Holt, Rinehart, and Winston, New York, 1965.

6. R. Gunning and H. Rossi, Analytic Functions of Several Complex Variables, PrenticeHall, Englewood Cliffs, N. J., 1965.

7. L. Hörmander, An Introduction to Complex Analysis in Several Variables, van Nostrand, Princeton, 1966.

8. J. Horváth, Topological Vector Spaces and Distributions, Addison-Wesley, Reading, Mass., 1966.

9. B. E. Johnson, Continuity of linear operators commuting with continuous linear operators, Trans. Amer. Math. Soc., 128 (1967), 88-102.

10. R. J. Loy, Local analytic structure in commutative F-algebras, (preprint).

11. E. A. Michael, Locally multiplicatively-convex topological algebras, Mem. Amer. Math. Soc., 11 (1952).

12. R. Narasimhan, Introduction to the Theory of Analytic Spaces, Springer-Verlag Lecture Notes 25 (1966).

13. C. E. Richart, Holomorphic convexity for general function algebras, Canad. J. Math., 20 (1968), 272-290.

3a. D. D. Clayton, A local characterization of analytic structure in a Banach algebra, Thesis, University of Utah, 1969.

Received August 30, 1972. Research for this paper was supported in part by NSF Grant GP 18729A1. 


\section{PACIFIC JOURNAL OF MATHEMATICS}

\section{EDITORS}

RICHARD ARENS (Managing Editor)

University of California

Los Angeles, California 90024

\section{R. A. Beaumont}

University of Washington

Seattle, Washington 98105

\section{J. DuGundJI*}

Department of Mathematics

University of Southern California

Los Angeles, California 90007

D. Gilbarg and J. Milgram

Stanford University

Stanford, California 94305

ASSOCIATE EDITORS
E. F. BECKENBACH
B. H. NeumanN
F. WOLF
K. YoSHIDA

\section{SUPPORTING INSTITUTIONS}

UNIVERSITY OF BRITISH COLUMBIA

CALIFORNIA INSTITUTE OF TECHNOLOGY

UNIVERSITY OF CALIFORNIA

MONTANA STATE UNIVERSITY

UNIVERSITY OF NEVADA

NEW MEXICO STATE UNIVERSITY

OREGON STATE UNIVERSITY

UNIVERSITY OF OREGON

OSAKA UNIVERSITY

\author{
UNIVERSITY OF SOUTHERN CALIFORNIA \\ STANFORD UNIVERSITY \\ UNIVERSITY OF TOKYO \\ UNIVERSITY OF UTAH \\ WASHINGTON STATE UNIVERSITY \\ UNIVERSITY OF WASHINGTON \\ AMERICAN MATHEMATICAL SOCIETY \\ NAVAL WEAPONS CENTER
}

The Supporting Institutions listed above contribute to the cost of publication of this Journal, but they are not owners or publishers and have no responsibility for its content or policies.

Mathematical papers intended for publication in the Pacific Journal of Mathematics should be in typed form or offset-reproduced, (not dittoed), double spaced with large margins. Underline Greek letters in red, German in green, and script in blue. The first paragraph or two must be capable of being used separately as a synopsis of the entire paper. Items of the bibliography should not be cited there unless absolutely necessary, in which case they must be identified by author and Journal, rather than by item number. Manuscripts, in duplicate if possible, may be sent to any one of the four editors. Please classify according to the scheme of Math. Rev. Index to Vol. 39. All other communications to the editors should be addressed to the managing editor, or Elaine Barth, University of California, Los Angeles, California, 90024.

50 reprints are provided free for each article; additional copies may be obtained at cost in multiples of 50 .

The Pacific Journal of Mathematics is issued monthly as of January 1966. Regular subscription rate: $\$ 48.00$ a year (6 Vols., 12 issues). Special rate: $\$ 24.00$ a year to individual members of supporting institutions.

Subscriptions, orders for back numbers, and changes of address should be sent to Pacific Journal of Mathematics, 103 Highland Boulevard, Berkeley, California, 94708.

\section{PUBLISHED BY PACIFIC JOURNAL OF MATHEMATICS, A NON-PROFIT CORPORATION}

Printed at Kokusai Bunken Insatsusha (International Academic Printing Co., Ltd.), 270, 3-chome Totsuka-cho, Shinjuku-ku, Tokyo 160, Japan.

* C. R. DePrima California Institute of Technology, Pasadena, CA 91109, will replace J. Dugundji until August 1974.

Copyright (C) 1973 by

Pacific Journal of Mathematics

All Rights Reserved 


\section{Pacific Journal of Mathematics}

\section{Vol. 49, No. $2 \quad$ June, 1973}

Wm. R. Allaway, On finding the distribution function for an orthogonal polynomial

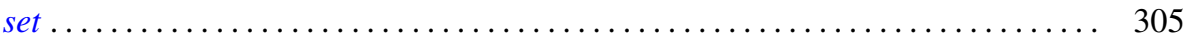

Eric Amar, Sur un théorème de Mooney relatif aux fonctions analytiques bornées... . 311

Robert Morgan Brooks, Analytic structure in the spectrum of a natural system . . . . 315

Bahattin Cengiz, On extremely regular function spaces . . . . . . . . . . . . . . 335

Kwang-nan Chow and Moses Glasner, Atoms on the Royden boundary . . . . . . . . . 339

Paul Frazier Duvall, Jr. and Jim Maxwell, Tame $Z^{2}$-actions on $E^{n} \ldots \ldots \ldots \ldots \ldots . .349$

Allen Roy Freedman, On the additivity theorem for $n$-dimensional asymptotic

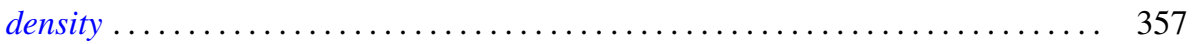

John Griffin and Kelly Denis McKennon, Multipliers and the group $L_{p}$-algebras . . . 365

Charles Lemuel Hagopian, Characterizations of $\lambda$ connected plane continua ....... 371

Jon Craig Helton, Bounds for products of interval functions . . . . . . . . . . . 377

Ikuko Kayashima, On relations between Nörlund and Riesz means . . . . . . . . . . 391

Everett Lee Lady, Slender rings and modules . . . . . . . . . . . . . . . . . . 397

Shozo Matsuura, On the Lu Qi-Keng conjecture and the Bergman representative

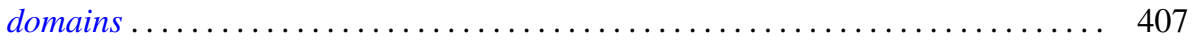

Stephen H. McCleary, The lattice-ordered group of automorphisms of an $\alpha$-set . . . 417

Stephen H. McCleary, o-2-transitive ordered permutation groups .......... 425

Stephen H. McCleary, o-primitive ordered permutation groups. II . . . . . . . . . 431

Richard Rochberg, Almost isometries of Banach spaces and moduli of planar

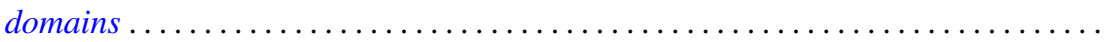

R. F. Rossa, Radical properties involving one-sided ideals . . . . . . . . . . . . . 467

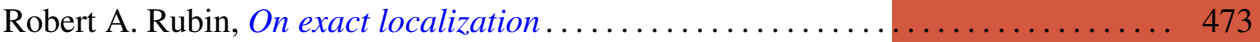

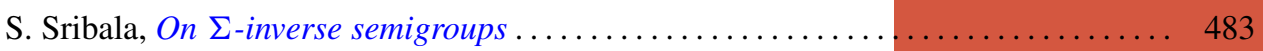

H. M. (Hari Mohan) Srivastava, On the Konhauser sets of biorthogonal polynomials suggested by the Laguerre polynomials ...................... 489

Stuart A. Steinberg, Rings of quotients of rings without nilpotent elements . ...... 493

Daniel Mullane Sunday, The self-equivalences of an $H$-space . . ............ 507

W. J. Thron and Richard Hawks Warren, On the lattice of proximities of $\check{C} e c h$

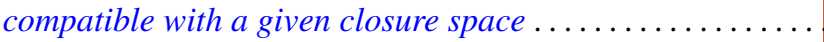

Frank Uhlig, The number of vectors jointly annihilated by two real quadratic forms determines the inertia of matrices in the associated pencil .

Frank Uhlig, On the maximal number of linearly independent real vectors annihilated simultaneously by two real quadratic forms ..............

Frank Uhlig, Definite and semidefinite matrices in a real symmetric matrix pencil . . 561

Arnold Lewis Villone, Self-adjoint extensions of symmetric differential operators . . . 569

Cary Webb, Tensor and direct products . ....................... 579

James Victor Whittaker, On normal subgroups of differentiable

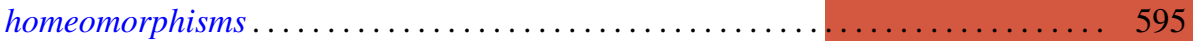

Jerome L. Paul, Addendum to: "Sequences of homeomorphisms which converge to

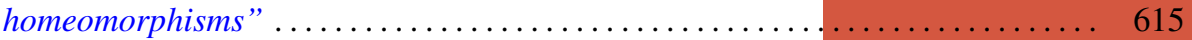

David E. Fields, Correction to: “Dimension theory in power series rings” ........ 616

Peter Michael Curran, Correction to: “Cohomology of finitely presented groups”. . . 617

Billy E. Rhoades, Correction to: “Commutants of some Hausdorff matrices” ...... 617

Charles W. Trigg, Corrections to: "Versum sequences in the binary system” ...... 619 